\title{
SPATIO-TEMPORAL CHANGES IN THE TROPHIC STRUCTURE OF ROCKY INTERTIDAL MOLLUSC ASSEMBLAGES ON A TROPICAL SHORE
}

\section{CAMBIOS ESPACIOTEMPORALES EN LA ESTRUCTURA TRÓFICA DE ASOCIACIONES DE MOLUSCOS DEL INTERMAREAL ROCOSO EN UN SUSTRATO TROPICAL}

\author{
C. Olabarria ${ }^{1}$ \\ J.L Carballo \\ C. Vega ${ }^{2}$ \\ ${ }^{1}$ Ecological Impacts of Coastal Cities \\ A Commonwealth Special Research Centre at the University of Sydney \\ Marine Ecology Laboratories, A11 \\ University of Sydney \\ Sydney, N.S.W. 2006, Australia \\ E-mail: colabarr@bio.usyd.edu.au \\ ${ }^{2}$ Instituto de Ciencias del Mar y Limnología \\ Universidad Nacional Autónoma de México \\ Av. Joel Montes Camarena s/n, apartado postal 811 \\ Mazatlán, C.P. 82000, México
}

Recibido en abril de 2000; aceptado en noviembre de 2000

\begin{abstract}
Patterns of the distribution and temporal variation of trophic groups within a benthic mollusc asemblage of a tropical shore (eastern tropical Pacific) were analysed in this study. Four intertidal rocky sites with different wave exposure were studied. A three-factor analysis of variance (fixed factors: trophic groups and level of exposure; random factor: site) and the Student-Newman-Keuls a posteriori test $(P=0.05)$ were applied to the biomass of trophic groups in order to know the variation among different sites. Carnivores, herbivores and suspension-feeders varied significantly among sites within each exposure level. Suspension-feeders were more abundant at exposed sites, while herbivores dominated in sheltered places. Biomass of herbivores was not correlated to macroalgal biomass. Total biomass was highest at exposed sites, increasing in winter and decreasing in summer. An ordination analysis carried out with the non-metric multidimensional scaling programme (MDS) revealed two trophic groups: groupA included samples from exposed sites (E1, E2) and group B included samples from sheltered sites (E3, E4). Graphic methods (ABC curves) detected changes along temporal and spatial scales, and these could be related to a high proportion of small species. Factors such as exposure level and type of substrate may control the distribution of different trophic groups.
\end{abstract}

Key words: molluscs, trophic structure, rocky intertidal, multivariant analyses, eastern tropical Pacific. 
Ciencias Marinas, Vol. 27, No. 2, 2001

\section{RESUMEN}

En este estudio se analizaron los patrones de distribución y variación temporal de los grupos tróficos de una asociación de moluscos en un sustrato tropical (Pacífico tropical oriental). Se estudiaron cuatro estaciones intermareales rocosas con diferente grado de exposición al oleaje. Se aplicó un análisis de varianza de tres factores (factores fijos: grupos tróficos y grado de exposición; factor al azar: sitio) y la prueba a posteriori de Student-Newman-Keuls $(P=0.05)$ con el objeto de conocer la variación entre las diferentes localidades. La biomasa de carnívoros, herbívoros y suspensívoros varió significativamente entre las distintas estaciones dentro de cada nivel de exposición. Los suspensívoros predominaron en áreas expuestas, mientras que los herbívoros dominaron en las estaciones protegidas. La biomasa de herbívoros no se correlacionó con la biomasa de macroalgas. La biomasa total fue mayor en lugares expuestos, mostrando un incremento en invierno y un descenso en verano. Un análisis de ordenación (MDS) reveló la existencia de dos grupos: el grupo A, que incluyó las muestras de los sitios expuestos (E1 y E2); y el grupo B, que incluyó las muestras de los sitios protegidos (E3 y E4). Los métodos gráficos (curvas $\mathrm{ABC}$ ) detectaron cambios espaciales y temporales que pudieron deberse a la alta proporción de especies pequeñas. Algunos factores como nivel de exposición y tipo de sustrato podrían haber controlado la distribución de los diferentes grupos.

Palabras clave: moluscos, estructura trófica, intermareal rocoso, análisis multivariantes, Pacífico tropical oriental.

\section{INTRODUCTION}

The complexity and variability of natural assemblages often make descriptions, interpretations and predictions of their structure difficult if not impossible. Although each species is thought to occupy a unique ecological niche, there are groups of species that commonly utilize certain resources of the environment in similar ways (i.e., functional groups). An understanding of these functional groups in the marine benthic assemblages gives an idea of how the dominant energy in the environment is used by the organisms and to what extent (Odum, 1971), and provides indirect information on the physical characteristics of the environment. The importance of the functional approach in an exploratory analysis of benthic communities has been emphasized by several authors (Warwick, 1982; Gagnon and Haedrich, 1991). The factors that control the distribution of trophic groups determine the structure of the community (Commito and Ambrose, 1985). The distribution and

\section{INTRODUCCIÓN}

La complejidad y variabilidad de las asociaciones naturales hacen difícil la interpretación, predicción y descripción de la estructura de los mismos. Aunque cada especie suele ocupar un nicho ecológico, hay grupos de especies que comúnmente utilizan ciertos recursos del ambiente de una manera similar (i.e., grupos funcionales). El conocimiento de estos grupos funcionales de las asociaciones bentónicas marinas nos da una idea de cómo y hasta que punto la energía dominante en el ecosistema es utilizada por los moluscos (Odum, 1971), y nos aporta información indirecta de las características físicas del medio ambiente. La importancia de una aproximación funcional en el análisis de las comunidades bentónicas ha sido enfatizada por varios autores (Warwick, 1982; Gagnon y Haedrich, 1991). Los factores que controlan la distribución de los distintos grupos tróficos determinan la estructura de la comunidad (Commito y Ambrose, 1985). La distribución y abundancia de los grupos funcionales se 
Olabarria et al.: Trophic structure of molluscs on a rocky shore in the tropical Pacific

abundance of functional groups are correlated with physical factors (Gray, 1974; Whitlatch and Obrebski, 1980; Cornet, 1985, 1986).

Factors governing the distribution of animals on intertidal rocky shores are commonly considered to follow the classical model of Connell (1961), in which the upper limits are set by physical variables, such as temperature or desiccation, and the lower limits by biological interactions, such as competition and predation. However, Underwood and Denley (1984) indicated that this generalization should not be used for intertidal organisms at all times and places. Thus, distribution patterns can be due to other factors, such as differences in settlement and/or recruitment (Dayton, 1975; Andrew and Choat, 1985), different mortality rates among different habitats (Andrew and Choat, 1985) and small-scale disturbances (Underwood and Chapman, 1999). Moreover, physical factors, such as different degrees of exposure to wave action (Lewis, 1964; Stephenson and Stephenson, 1972), different temperature regimes and different kinds of rocky substrates, influence the trophic structure and play an important role in the energy flow through the communities (McQuaid and Branch, 1985). The role of physical factors (e.g., wave exposure) is really important, since the impact of biotic forces (e.g., recruitment, predation, competition, grazing) can vary along these environmental gradients (Polis et al., 1996).

This paper describes patterns of spatiotemporal variability of intertidal mollusc assemblages in terms of functional groups that experienced different environmental conditions (exposed versus sheltered). Graphical and multivariate methods were used to determine different assemblages. The data were used to test the model that, as has been described for intertidal assemblages: one of the physical factors structuring rocky intertidal assemblages is the exposure to wave action. correlacionan con los factores físicos del ambiente. (Gray, 1974; Whitlatch y Obrebski, 1980; Cornet, 1985, 1986).

Algunos de los factores que ocasionan la distribución de los organismos en sustratos rocosos intermareales parecen seguir el modelo clásico propuesto por Connell (1961), en el cual el límite superior está marcado por variables físicas, como temperatura y desecación, y el límite inferior por interacciones biológicas, como competencia y predación. Sin embargo, Underwood y Denley (1984) indicaron que esta generalización no se puede aplicar a todos los organismos intermareales en todos los momentos y lugares. Así, los patrones de distribución pueden deberse a otros factores, tales como diferencias en asentamiento y/o reclutamiento (Dayton, 1975; Andrew y Choat, 1985), diferentes tasas de mortalidad entre hábitats diferentes (Andrew y Choat, 1985) y perturbaciones a pequeña escala (Underwood y Chapman, 1999). Asimismo, factores físicos, tales como diferentes grados de exposición al oleaje (Lewis, 1964; Stephenson y Stephenson, 1972), diferentes regímenes de temperatura y diferentes sustratos rocosos, influyen en la estructura trófica y juegan un papel muy importante en el flujo energético en las comunidades (MacQuaid y Branch, 1985). También, el papel de los factores físicos (e.g., exposición al oleaje) es muy importante, ya que los procesos biológicos (e.g., reclutamiento, predación, competencia, herbivorismo) pueden variar a lo largo de gradientes ambientales (Polis et al., 1996).

Este trabajo describe los patrones de variabilidad espaciotemporal de asociaciones intermareales de moluscos en términos de grupos funcionales bajo diferentes condiciones ambientales (expuesto contra protegido). Los métodos gráficos y multivariantes se usaron para determinar las diferentes asociaciones. Los datos se usaron para probar el modelo que, como ha sido descrito para asociaciones 
Ciencias Marinas, Vol. 27, No. 2, 2001

\section{MATERIALS AND METHODS}

Four intertidal sites in Mazatlán Bay (eastern tropical Pacific) were sampled every three months during 1998. The study area was within a $15-\mathrm{km}$ zone (fig. 1). Stations E1 and E2 were close to the town, whereas stations E3 and E4 were not (at a distance of approximately $15 \mathrm{~km}$ ). The first two stations were in exposed rocky areas, while E3 and E4 were sheltered and close to a sand shelf. Sites E2 and E4 received moderate loads of coarse sands resuspended by water turbulence in winter; the latter was a sloping, sandy-rocky platform covered with seaweed. Quantitative mollusc samples were collected according to previous minimum area calculations (Estacio et al., 1999). The minimum area was calculated in four quadrants of $0.25 \mathrm{~m}^{2}$ sampled from mid to low shore. Three replicates were taken at each sampling station, so that a total surface of $3 \mathrm{~m}^{2}$ (12 samples) was sampled at each site. Samples were taken by scraping the entire surface of the quadrant with a spatula and separated using a $0.5-\mathrm{mm}$ mesh. The material was preserved in $70 \%$ ethanol.

The specimens were identified to species, counted and weighed; the biomass of each species was determined and is expressed herein as blotted wet weight. Molluscs were not decalcified, because the material was required for subsequent taxonomic work (Warwick and Ruswahyuni, 1987). Although there is still considerable uncertainty about exact feeding habits and diets of many species (Walker and Rees, 1979; Gray, 1981), each of the 90 mollusc species sampled was assigned to a trophic guild, following Keen (1971) and Reguero et al. (1991, 1993). Organisms were ranked into six trophic groups: suspension-feeders, herbivores, deposit-feeders, carnivores, parasites and omnivores. The dominant species, those occurring in more than $10 \%$ of the samples, are represented in table 1 . intermareales: uno de los factores físicos que estructura las asociaciones intermareales rocosas es el grado de exposición al oleaje.

\section{MATERIALES Y MÉTODOS}

Cuatro estaciones en la Bahía de Mazatlán (Pacífico tropical oriental) fueron muestreadas trimestralmente durante 1998. La zona de estudio abarcó $15 \mathrm{~km}$ (fig. 1). Los sitios E1 y E2 se localizaron en la proximidad de la ciudad, mientras que los sitios E3 y E4 se localizaron lejos (a unos $15 \mathrm{~km}$ de distancia). Las dos primeras estaciones fueron zonas rocosas expuestas, mientras que E3 y E4 se localizaron en una zona protegida próxima a una plataforma arenosa. Los sitios E2 y E4 recibieron aportes moderados de arena puesta en suspensión por la turbulencia de agua durante el invierno. El sitio E4 fue una plataforma areno-rocosa cubierta por algas. Se tomaron muestras cuantitativas de moluscos según el previo cálculo del área mínima cuantitativa (Estacio et al., 1999). El área mínima calculada fueron cuatro cuadrados de $0.25 \mathrm{~m}$ de lado, tomados desde la zona media de marea hasta la zona inferior de marea. Se tomaron tres réplicas en cada sitio de muestreo, de forma que una superficie total de $3 \mathrm{~m}^{2}$ (12 muestras) fue muestreada en cada sitio. Las muestras se obtuvieron raspando toda la superficie del cuadrado con una espátula. Posteriormente, el material recolectado fue llevado al laboratorio y separado en una malla de $0.5 \mathrm{~mm}$, siendo conservado finalmente en alcohol al $70 \%$.

Los especímenes fueron separados, contados e identificados a nivel de especie, y cada especie fue pesada. Se determinó el peso húmedo y peso seco para cada especie. Los moluscos no fueron descalcificados, puesto que el material fue necesario para posteriores trabajos taxonómicos (Warwick y Ruswahyuni, 1987). Aunque existe una considerable incertidumbre acerca de los hábitos tróficos y dietas 


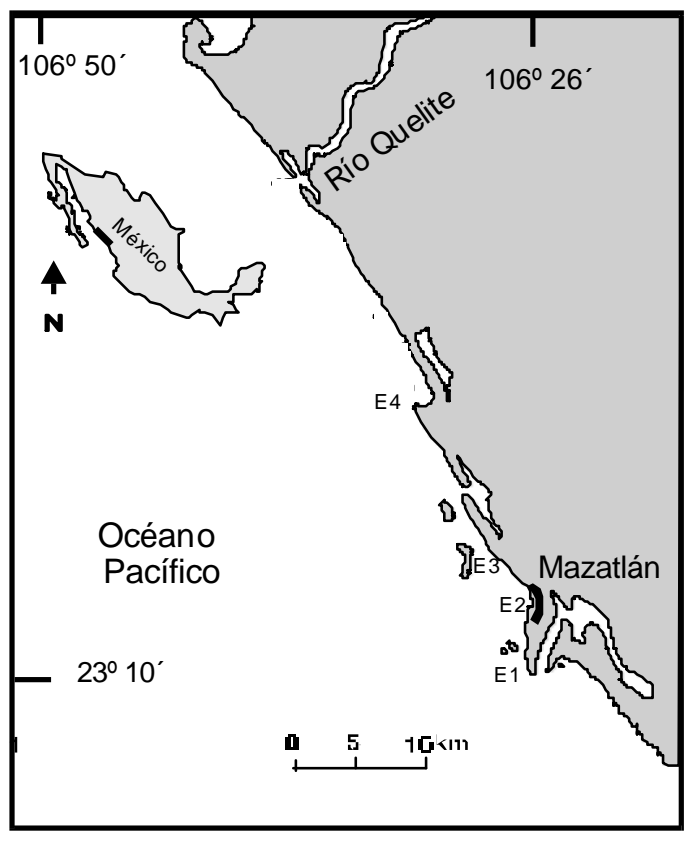

Figure 1. Location of the study area in Mazatlán Bay.

Figura 1. Localización del área de estudio en la Bahía de Mazatlán.

Data of macroalgal biomass at each site and time were determined in order to establish a relationship between grazers and algal availability.

Spatial variation of biomass for each trophic group was tested through a three-factor analysis of variance. These factors were: the trophic group (fixed factor with six levels: carnivores, suspension-feeders, deposit-feeders, parasites, herbivores and omnivores, orthogonal), level of exposure (fixed factor with two levels: sheltered and exposed, orthogonal), and site (random factor with four levels: E1, E2, E3 and E4, nested within exposure level). Previously, normality (Kolmogorov-Smirnov test) and homogeneity of variance (Cochran test) were verified. When there were significant departures from homogeneity, the data were double square-root transformed, which de muchas especies (Walker y Rees, 1979; Gray, 1981), cada una de las 90 especies de moluscos fue asignada a un grupo trófico, según Keen (1971) y Reguero et al. (1991, 1993). Los organismos fueron clasificados en seis grupos tróficos: suspensívoros, herbívoros, depositívoros, carnívoros, parásitos y omnívoros. Las especies más abundantes que aparecieron en más del $10 \%$ de las muestras aparecen representadas en la tabla 1 .

Se tomaron datos de biomasa de macroalgas en cada sitio y época, con el objeto de conocer posibles relaciones entre la distribución de herbívoros y algas.

La posible variación espacial en la biomasa fue analizada a través de un análisis de varianza con tres factores. Estos factores fueron: grupo trófico (factor fijo con seis niveles: carnívoros, suspensívoros, depositívoros, parásitos, 
Table 1. Mollusc species found in more than $10 \%$ of the samples, listed in order of importance and categorized by trophic groups (Keen, 1971; Reguero et al., 1991, 1993).

Tabla 1. Especies de moluscos que aparecieron en más del $10 \%$ de las muestras, ordenadas según su importancia y categorizadas por grupos tróficos (Keen, 1971; Reguero et al., 1991, 1993).

\begin{tabular}{|c|c|}
\hline Species & Trophic group \\
\hline 1. Fartulum sp. Carpenter & Deposit-feeder \\
\hline 2. Barleeia alderi (Carpenter, 1857) & Herbivore \\
\hline 3. Cerithium mekei Carpenter, 1857 & Herbivore \\
\hline 4. Siphonaria maura Sowerby, 1832 & Herbivore \\
\hline 5. Eulithidium perforata (Philippi, 1848) & Herbivore \\
\hline 6. Parviturbo concepsionensis (Lowe, 1932) & Omnivore \\
\hline 7. Parvianachis pygmaea (Sowerby, 1832) & Carnivore \\
\hline 8. Rissoella excolpa Bartsch, 1920 & Deposit-feeder \\
\hline 9. Lottia mitella (Menke, 1847) & Herbivore \\
\hline 10. Pteria sterna (Gould, 1851) & Suspension-feeder \\
\hline 11. Stramonita haemostoma (Linnaeus, 1758) & Carnivore \\
\hline 12. Chrysallida vizcainoana (Baker, Hanna and Strong, 1926) & Parasite \\
\hline 13. Cardita laticostata Sowerby, 1833 & Suspension-feeder \\
\hline 14. Caecum compactum Carpenter, 1857 & Deposit-feeder \\
\hline 15. Lithophaga attenuata rogersi Berry, 1957 & Suspension-feeder \\
\hline 16. Tegula ligulata (Menke, 1850) & Herbivore \\
\hline 17. Barbatia bailyi (Bartsch, 1931) & Suspension-feeder \\
\hline 18. Crepidula aculeata (Gmelin, 1791) & Suspension-feeder \\
\hline 19. Fissurella microtrema Sowerby, 1835 & Herbivore \\
\hline 20. Chama mexicana Carpenter, 1857 & Suspension-feeder \\
\hline 21. Fissurella nigrocincta Carpenter, 1856 & Herbivore \\
\hline 22. Hiatella artica (Linnaeus, 1767) & Suspension-feeder \\
\hline 23. Tectura fascicularis (Menke, 1851) & Herbivore \\
\hline 24. Radsiella muscaria (Reeve, 1848) & Herbivore \\
\hline 25. Lottia standfordiana (Berry, 1957) & Herbivore \\
\hline 26. Choromytilus palliopunctatus (Carpenter, 1857) & Suspension-feeder \\
\hline 27. Fissurella virescens Sowerby, 1835 & Herbivore \\
\hline
\end{tabular}


generally resulted in homoscedasticity. Factors and their interactions were further analysed using the Student-Newman-Keuls test to determine which values were significantly different at $P<0.05$ (Day and Quinn, 1989).

Abundance/biomass curves were used to establish possible changes in assemblages (Warwick et al., 1987). To compare the similarity between sites and times, the ordination techniques recommended by Field et al.(1982) were followed. The similarity matrix was established using double square-root transformed biomass data and the Bray-Curtis measure of similarity (Bray and Curtis, 1957). An ordination analysis (nMDS) was used to determine the assemblages of different trophic groups. The Kruskal stress coefficient was used in order to test the ordination obtained by the MDS (Clarke, 1990).

\section{RESULTS}

\section{Trophic structure of intertidal communities}

Suspension-feeders $(40.58 \%)$ were the dominant trophic group in this study, followed by carnivores (33.23\%) and herbivores $(35.38 \%)$, while parasites were the smallest group $(0.045 \%)$. Sites E1 and E2 showed the highest biomass of suspension-feeders, while sites E3 and E4 were dominated by herbivores and carnivores, respectively (table 2). Total biomass of trophic groups was highest at site $\mathrm{E} 1$, with a maximum in winter and a minimum in autumn. The ANOVA analysis showed significant differences among trophic groups at the different sites depending on the wave exposure $\left(F_{30,240}: 3.97, P<0.0001\right)$ (table 3$)$. The Student-Newman-Keuls test showed significant differences in carnivore distribution between sites E1 and E3, E1 and E4, and E1 and E2 $(P<0.05)$; in herbivore distribution between E1 and E3, E2 and E3, and E3 and E4 $(P<0.05)$; and in suspension-feeder herbívoros y omnívoros, ortogonal), grado de exposición (factor fijo con dos niveles: protegido y expuesto, ortogonal) y sitio (factor al azar con cuatro niveles: E1, E2, E3 y E4, anidado en factor grado de exposición). Previamente, se verificó la normalidad (prueba de Kolmogorov-Smirnov) y la homogeneidad de varianzas (prueba de Cochran). Los datos fueron transformados mediante raíz cuarta en aquellos casos donde las varianzas no fueron homogéneas. Los factores e interacciones que fueron significativos en el análisis de varianza fueron analizados posteriormente a través de la prueba de Student-Newman-Keuls, con el objeto de determinar los sitios en los que los grupos tróficos fueron significativamente diferentes a $P<0.05$ (Day y Quinn, 1989).

Se construyeron las curvas de abundancia/ biomasa $(\mathrm{ABC})$ con el objeto de encontrar posibles cambios en las asociaciones. Para comparar la similitud entre estaciones y épocas en términos de grupo trófico, se siguieron las técnicas de ordenación recomendadas por Field et al. (1982). La matriz de similitud se construyó transformando los datos de biomasa con la raíz cuarta y analizando los datos a través de la medida de similitud de Bray-Curtis (Bray y Curtis, 1957). Un análisis de ordenación (nMDS) se usó para determinar las asociaciones de los diferentes grupos tróficos. El coeficiente de estrés de Kruskal fue utilizado para comprobar la ordenación obtenida (Clarke, 1990).

\section{RESULTADOS}

\section{Estructura trófica de las asociaciones intermareales}

Los suspensívoros (40.58\%) fueron el grupo trófico dominante en este estudio, seguido de los carnívoros $(33.23 \%)$ y herbívoros $(35.38 \%)$, mientras que los parásitos fueron el grupo más pequeño $(0.045 \%)$. Los sitios E1 
Ciencias Marinas, Vol. 27, No. 2, 2001

Table 2. Mean biomass (blotted weight, $\mathrm{g} \mathrm{m}^{-2}$ ) of the trophic groups and macroalgae in winter, spring, summer and autumn at the four sites (E1, E2, E3 and E4).

Tabla 2. Biomasa media (peso seco, $\mathrm{g} \mathrm{m}^{-2}$ ) de los grupos tróficos y macroalgas en invierno, primavera, verano y otoño en las cuatro estaciones (E1, E2, E3 y E4).

\begin{tabular}{|c|c|c|c|c|}
\hline & Winter & Spring & Summer & Autumn \\
\hline \multicolumn{5}{|l|}{$\overline{\mathbf{E 1}}$} \\
\hline Carnivores & $50.81 \pm 10.22$ & $65.357 \pm 22.21$ & $18.26 \pm 5.43$ & $7.15 \pm 2.54$ \\
\hline Deposit-feeders & $0.39 \pm 0.11$ & $0.38 \pm 0.02$ & $0.03 \pm 0.01$ & $0.38 \pm 0.01$ \\
\hline Suspension-feeders & $105.95 \pm 34.22$ & $26.26 \pm 10.21$ & $0.35 \pm 0.11$ & $29.39 \pm 12.54$ \\
\hline Parasites & $0.03 \pm 0.01$ & $0.02 \pm 0.01$ & $0.22 \pm 0.12$ & $0.13 \pm 0.05$ \\
\hline Omnivores & $0.32 \pm 0.11$ & $0.36 \pm 0.03$ & $0.04 \pm 0.01$ & $0.33 \pm 0.25$ \\
\hline Herbivores & $3.25 \pm 1.02$ & $4.45 \pm 1.34$ & $0.92 \pm 0.05$ & $7.97 \pm 1.54$ \\
\hline Macroalgae & $140.62 \pm 14.22$ & $166.04 \pm 32.31$ & $233.45 \pm 34.78$ & $146.53 \pm 12.98$ \\
\hline \multicolumn{5}{|l|}{ E2 } \\
\hline Carnivores & $7.45 \pm 3.13$ & $0.73 \pm 0.02$ & $0.02 \pm 0.01$ & $0.61 \pm 0.22$ \\
\hline Deposit-feeders & $0.484 \pm 0.23$ & $0.05 \pm 0.02$ & $0.03 \pm 0.01$ & $0.16 \pm 0.02$ \\
\hline Suspension-feeders & $5.41 \pm 2.13$ & $6.77 \pm 2.34$ & $1.60 \pm 0.41$ & $14.86 \pm 5.22$ \\
\hline Parasites & $0.07 \pm 0.01$ & $0.22 \pm 0.02$ & 0.00 & $0.03 \pm 0.02$ \\
\hline Omnivores & $0.33 \pm 0.02$ & $0.03 \pm 0.02$ & $0.03 \pm 0.01$ & $0.10 \pm 0.02$ \\
\hline Herbivores & $14.32 \pm 3.13$ & $1.75 \pm 0.02$ & $0.35 \pm 0.01$ & $8.23 \pm 2.22$ \\
\hline Macroalgae & $145.10 \pm 44.78$ & $172.56 \pm 56.76$ & $157.89 \pm 23.01$ & $211.98 \pm 56.90$ \\
\hline \multicolumn{5}{|l|}{$\mathbf{E 3}$} \\
\hline Carnivores & $4.32 \pm 1.34$ & $3.27 \pm 0.89$ & $14.79 \pm 3.67$ & $2.31 \pm 1.23$ \\
\hline Deposit-feeders & $0.03 \pm 0.01$ & $0.18 \pm 0.09$ & $0.24 \pm 0.12$ & $0.04 \pm 0.01$ \\
\hline Suspension-feeders & $3.52 \pm 0.45$ & $8.81 \pm 1.32$ & $0.11 \pm 0.04$ & $20.62 \pm 11.23$ \\
\hline Parasites & $0.03 \pm 0.02$ & $0.02 \pm 0.01$ & $0.01 \pm 0.00$ & $0.31 \pm 0.15$ \\
\hline Omnivores & 0.00 & $0.39 \pm 0.11$ & $0.02 \pm 0.01$ & 0.00 \\
\hline Herbivores & $37.61 \pm 11.34$ & $20.91 \pm 4.89$ & $17.51 \pm 3.67$ & $12.56 \pm 5.34$ \\
\hline Macroalgae & $125.67 \pm 33.42$ & $96.33 \pm 10.89$ & $99.78 \pm 13.67$ & $132.69 \pm 67.8$ \\
\hline \multicolumn{5}{|l|}{$\mathbf{E 4}$} \\
\hline Carnivores & $2.97 \pm 1.35$ & $7.10 \pm 2.34$ & $3.45 \pm 2.22$ & $0.50 \pm 0.01$ \\
\hline Deposit-feeders & $0.23 \pm 0.01$ & $0.21 \pm 0.02$ & $0.24 \pm 0.01$ & $0.03 \pm 0.01$ \\
\hline Suspension-feeders & $4.20 \pm 1.35$ & $0.21 \pm 0.04$ & $1.65 \pm 0.55$ & $4.49 \pm 2.12$ \\
\hline Parasites & $0.22 \pm 0.12$ & $0.34 \pm 0.03$ & $0.06 \pm 0.02$ & $0.44 \pm 0.01$ \\
\hline Omnivores & $0.13 \pm 0.03$ & $0.34 \pm 0.02$ & $0.18 \pm 0.03$ & $0.29 \pm 0.01$ \\
\hline Herbivores & $3.54 \pm 1.35$ & $1.50 \pm 0.33$ & $1.96 \pm 0.67$ & $3.25 \pm 0.01$ \\
\hline Macroalgae & $6.80 \pm 1.35$ & $128.37 \pm 52.34$ & $109.26 \pm 32.22$ & $145.18 \pm 34.01$ \\
\hline
\end{tabular}


Olabarria et al.: Trophic structure of molluscs on a rocky shore in the tropical Pacific

Table 3. Three-factor analysis of variance (trophic group and exposition to wave are fixed factors, site is a random factor). $\mathrm{Tr}=$ trophic groups, ex $=$ exposition to wave and $\mathrm{si}=$ site.

Tabla 3. Análisis de varianza de tres factores (grupo trófico y grado de exposición son factores fijos, sitio es un factor al azar). $\mathrm{Tr}=$ grupo trófico, ex = grado de exposición y si = sitio.

\begin{tabular}{lrrrrl}
\hline Source of variation & d.f. & Mean square & $F$ & $P$ & $F$ versus \\
\hline $\operatorname{Tr}$ & 5 & 1086.1389 & 2.90 & $<0.05$ & $\operatorname{Tr} \times$ si (ex) \\
Ex & 1 & 1270.8655 & 0.79 & $>0.05$ & $\mathrm{Si}(\mathrm{ex})$ \\
$\mathrm{Si}(\mathrm{ex})$ & 6 & 1607.8422 & 8.88 & $<0.0001$ & $\mathrm{Res}$ \\
$\operatorname{Tr} \times \mathrm{ex}$ & 5 & 1084.7934 & 1.51 & $>0.05$ & $\operatorname{Tr} \times$ si (ex) \\
$\operatorname{Tr} \times \operatorname{si}(\mathrm{ex})$ & 30 & 719.1740 & 3.97 & $<0.0001$ & $\operatorname{Res}$ \\
$\operatorname{Res}$ & 240 & 181.1127 & & & \\
Total & 287 & & & & \\
\hline
\end{tabular}

distribution between $\mathrm{E} 1$ and $\mathrm{E} 2, \mathrm{E} 1$ and E3, and $\mathrm{E} 1$ and $\mathrm{E} 4(P<0.05)$. There was no correlation between macroalgal biomass and herbivore biomass distribution $(R=0.13, F=0.24$, d.f. $=1.14, P=0.665)$. Algae in particular were abundant at E2 and E1, whereas the highest herbivore biomass occurred at site E3. Algae and herbivores were particularly scarce at E4 (table 2). On the other hand, there was a recurrent temporal pattern in distribution of macroalgal and herbivore biomass, since the former peaked in autum except at site E1, while the latter reached the maximum in winter except at E1.

Suspension-feeders were dominant at site E1, followed by carnivores (fig. 2). The suspension-feeder Chama mexicana Carpenter (64.76 $\mathrm{g} \mathrm{m}^{2}$ ) accounted for almost $44 \%$ of the mean annual biomass of the group in this assemblage. Stramonita haemastoma (Linnaeus) contributed up to $84.69 \%$ of the carnivore group biomass. Herbivore biomass was distributed amongst several species, whereas depositivore biomass was mainly represented by Fartulum sp.

Suspension-feeders were the dominant group at E2, followed by herbivores (fig. 2). y E2 tuvieron la mayor biomasa de suspensívoros, mientras que en los sitios E3 y E4 dominaron los herbívoros y carnívoros (tabla 2). La biomasa total fue más alta en el sitio E1, con un máximo en invierno y un mínimo en otoño. El análisis de varianza mostró diferencias significativas en la distribución de los grupos tróficos entre los diferentes sitios dependiendo del nivel de exposición $\left(F_{30,240}: 3.97, P<0.0001\right)$ (tabla 3). La prueba de Student-Newman-Keuls reveló diferencias significativas en la distribución de carnívoros entre los sitios E1 y E3, E1 y E4, y E1 y E2 $(P<0.05)$; en la distribución de herbívoros entre los sitios E1 y E3, E2 y E3, y E3 y E4 $(P<0.05)$; y en la distribución de suspensívoros entre los sitios E1 y E2, E1 y E3, y E1 y E4 $(P<0.05)$. No hubo correlación entre la distribución de biomasa de macroalgas y la de herbívoros $(R=0.13, F=0.24$, g.l.=1.14, $P=0.665)$. Las algas fueron particularmente abundantes en los sitios E2 y E1, mientras que la mayor biomasa de herbívoros se dio en el sitio E3. Las algas y herbívoros fueron particularmente escasos en el sitio E4 (tabla 2). Por otro lado, un patrón temporal recurrente pudo observarse en la distribución de biomasa de macroalgas y herbívoros, puesto 


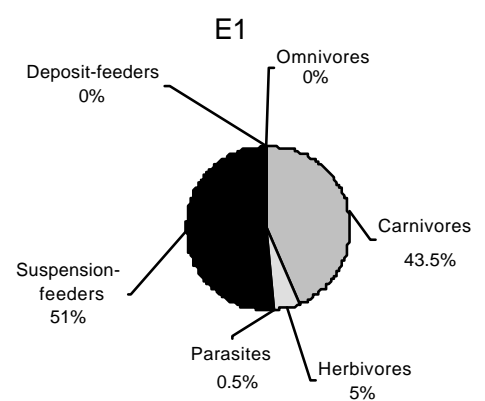

E3

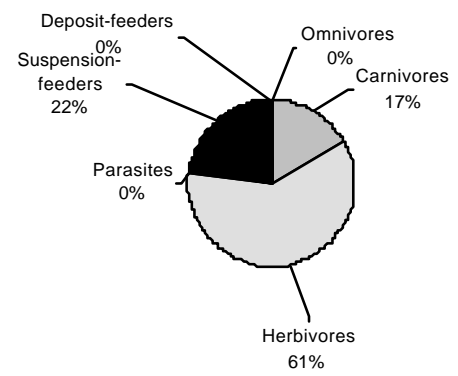

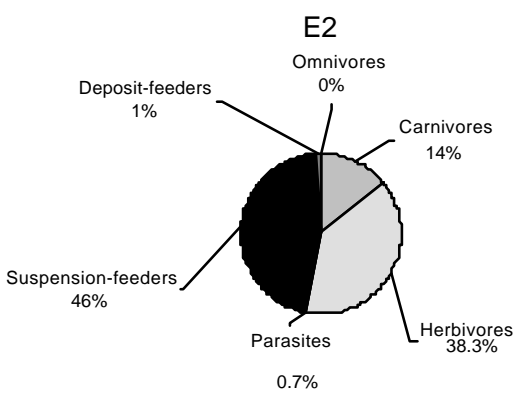

E4

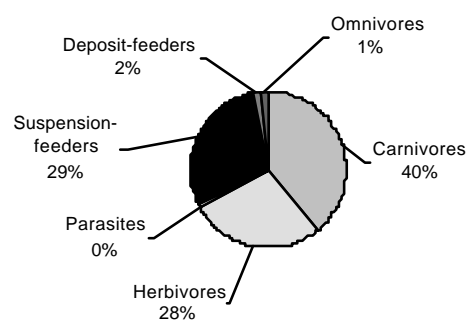

Figure 2. Total percentage of biomass (wet weight, $\mathrm{g} \mathrm{m}^{-2}$ ) of each trophic group at the four sites.

Figura 2. Porcentaje total de biomasa (peso húmedo, $\mathrm{g} \mathrm{m}^{-2}$ ) de cada grupo trófico en los cuatro sitios.

The suspension-feeders Chama mexicana, Choromytilus palliopunctatus (Carpenter) and Pteria sterna (Gould) (30.53 $\left.\mathrm{g} \mathrm{m}^{-2}\right)$ represented up to $48.92 \%$ of the total biomass of the assemblage. The herbivore Cerithium menkei Carpenter accounted for $38.87 \%$ of the mean annual biomass of this group at this site.

Herbivores predominated at E3, accounting for $61 \%$ of the mean annual biomass (fig. 2). Hipponix pilosus Gould, Cerithium menkei and Cerithium sp. Bruguière $\left(53.75 \mathrm{~g} \mathrm{~m}^{2}\right)$ accounted for $36.61 \%$ of the mean annual biomass. Suspension-feeders represented $22 \%$ of the mean annual biomass. Chama mexicana was the main suspension-feeder $(34.5 \%$ of the total biomass).

Carnivores were dominant at E4, followed by suspension-feeders and herbivores (fig. 2). que aquellas alcanzaron un pico en otoño y éstos en invierno excepto en el sitio E1.

Los suspensívoros fueron el grupo dominante en el sitio E1, seguido de los carnívoros (fig. 2). El suspensívoro Chama mexicana Carpenter (64.76 $\mathrm{g} \mathrm{m}^{-2}$ ) representó casi el $44 \%$ de la biomasa media anual del grupo en esta asociación. Stramonita haemastoma (Linnaeus) contribuyó con el $84.69 \%$ de la biomasa en el grupo de los carnívoros. Varias especies contribuyeron ampliamente a la biomasa de herbívoros, mientras que la biomasa de depositívoros estuvo representada principalmente por Fartulum sp.

Los suspensívoros fueron el grupo dominante en el sitio E2, seguido de los herbívoros (fig. 2). Las especies suspensívoras Chama mexicana, Choromytilus 
Olabarria et al.: Trophic structure of molluscs on a rocky shore in the tropical Pacific

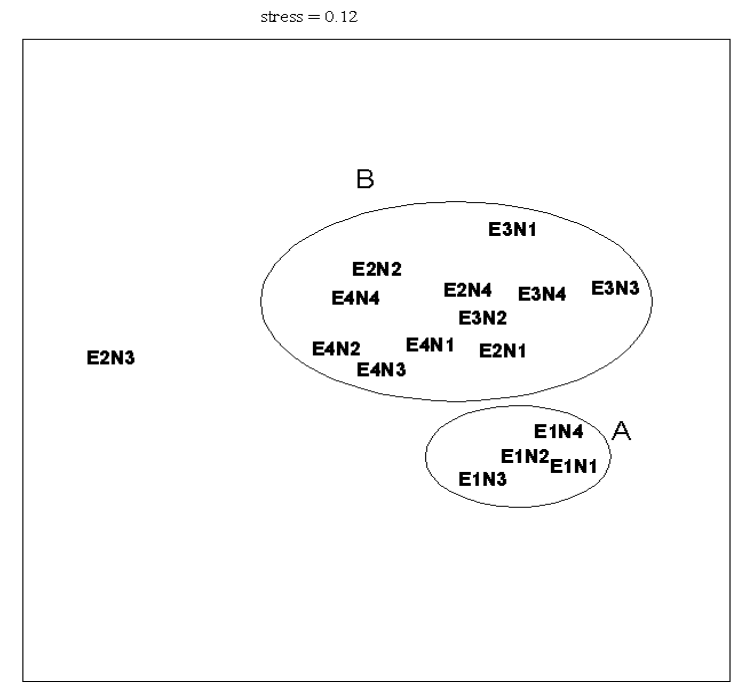

Figure 3. Multi-dimensional scaling (MDS) ordination of 16 samples from sites E1, E2, E3 and E4. This analysis was based on Bray-Curtis similarities among sites E1, E2, E3 and E4 in winter, spring, summer and autumn. N1, winter samples; N2, spring samples; N3, summer samples; and N4, autumn samples.

Figura 3. Ordenación multidimensional (MDS) de las 16 muestras de los sitios E1, E2, E3 y E4. Este análisis se basó en las similitudes de Bray-Curtis entre los sitios E1, E2, E3 y E4 en invierno, primavera, verano y otoño. N1, muestras de invierno; N2, muestras de primavera; N3, muestras de verano; y N4, muestras de otoño.

The carnivorous species Stramonita haemastoma and Mancinella speciosa (Valenciennes) $\left(10.57 \mathrm{~g} \mathrm{~m}^{-2}\right.$ ) were the main contributors to biomass in this group. Chama mexicana accounted for $48.93 \%$ of the mean annual biomass of the suspension-feeders. Siphonaria maura Sowerby and Tectura fascicularis (Menke) were the most important contributors in the herbivorous group. The rest of the trophic groups were similarly represented (fig. 2).

The MDS analysis (stress $=0.12$ ) revealed two groups: group $\mathrm{A}$ at sites E1 and E2, and group B at sites E3 and E4 (fig. 3). In group A, most of the biomass was made up of suspensivores and carnivores, with very few herbivores. In group $\mathrm{B}$, however, the herbivorous species accounted for more than $25 \%$ of the biomass. The E2 summer sample was isolated palliopunctatus (Carpenter) y Pteria sterna (Gould) (30.53 $\mathrm{g} \mathrm{m}^{-2}$ ) fueron las que contribuyeron con un $48.92 \%$ del total de la biomasa en la asociación. El herbívoro Cerithium menkei Carpenter representó el $38.87 \%$ de la media anual de la biomasa de este grupo en este sitio.

Los herbívoros predominaron en el sitio E3, representando el $61 \%$ de la media anual de la biomasa (fig. 2). Hipponix pilosus Gould, Cerithium menkei y Cerithium sp. Bruguière $\left(53.75 \mathrm{~g} \mathrm{~m}^{-2}\right.$ ) contribuyeron con el $36.61 \%$ de la biomasa media anual en este sitio. Los suspensívoros fueron importantes, representando el $22 \%$ de la biomasa media anual. Chama mexicana fue la especie más importante (34.5\% del total de la biomasa).

Los carnívoros fueron el grupo dominante en el sitio E4, seguido de los suspensívoros y herbívoros (fig. 2). Las especies Stramonita 
Ciencias Marinas, Vol. 27, No. 2, 2001

since all the trophic categories were poorly represented in it.

\section{Trophic dynamics and abundance/biomass curves}

The herbivores and suspension-feeders had the lowest biomass values in summer (table 2). A tendency of biomass to increase in winter and to decrease in summer was observed. This temporal pattern was nearly the same for deposit-feeders (e.g., Fartulum sp. was more abundant in winter because of sediment accumulation provoked by water turbulence), parasites and omnivores; however, herbivores peaked in summer. On the other hand, carnivores presented the lowest biomass value in spring, whereas herbivore and suspensionfeeder biomass values were lowest in autumn (table 2).

While the MDS analysis provided a good clustering, the contribution of single species to assemblages can be better explored through dominance curves. The ABC method showed changes at sites E2, E3 and E4, while E1 did not vary along a temporal scale (fig. 4A-D). The curves showed a change in the trophic structure at E2 in winter, which stabilized in spring and autumn (fig. 4E-H). Site E3 presented an unstable situation over the year (fig. 5A-D). Site E4 was very unstable in winter, stabilizing somewhat towards spring, and changing again in summer and throughout the autumn (fig. 5E-H).

\section{DISCUSSION}

\section{Spatial trophic structure}

The study of the trophic structure suggested a clear spatial distribution in Mazatlán Bay, with two main assemblages. The trophic structure of the assemblages at each sampling site seemed to be defined by a series of local haemastoma y Mancinella speciosa (Valenciennes) (10.57 $\mathrm{g} \mathrm{m}^{-2}$ ) fueron las que principalmente contribuyeron a la biomasa de este grupo. Chama mexicana representó el $48.93 \%$ de la biomasa media anual en el grupo de los suspensívoros. Siphonaria maura Sowerby y Tectura fascicularis (Menke) fueron las especies más importantes dentro de los herbívoros. El resto de los grupos tróficos estuvo similarmente representado (fig. 2).

El análisis MDS (estrés $=0.12$ ) reveló dos grupos: el grupo A, que incluyó las muestras del sitio E1 y E2; y el grupo B, que estuvo formado por las muestras de los sitios E3 y E4 (fig. 3). En el grupo A dominaron los suspensívoros y carnívoros, con escasos herbívoros. En el grupo B, sin embargo, los herbívoros representaron más del 25\% de la biomasa. La muestra de verano en E2 se separó de los dos grupos, ya que todas las categorías tróficas estuvieron escasamente representadas en dicha muestra.

\section{Dinámica trófica y curvas de abundancia/biomasa}

Los herbívoros y suspensívoros tuvieron la biomasa más baja en verano (tabla 2). Se observó una tendencia de la biomasa a incrementarse en invierno y disminuir en verano. Este patrón temporal fue similar para los depositívoros (e.g., Fartulum sp. fue más abundante en invierno debido a la acumulación de sedimento originada por la turbulencia del agua), parásitos y omnívoros; sin embargo, los herbívoros presentaron un pico en verano. Por otro lado, los carnívoros presentaron la biomasa más baja en primavera, mientras que los herbívoros y suspensívoros alcanzaron los valores más bajos en otoño (table 2).

Aunque el análisis MDS presentó un buen agrupamiento, la contribución de cada especie a las distintas asociaciones puede estudiarse mejor a través de las curvas de dominancia. El método $\mathrm{ABC}$ mostró cambios en los sitios E2, 
Olabarria et al.: Trophic structure of molluscs on a rocky shore in the tropical Pacific
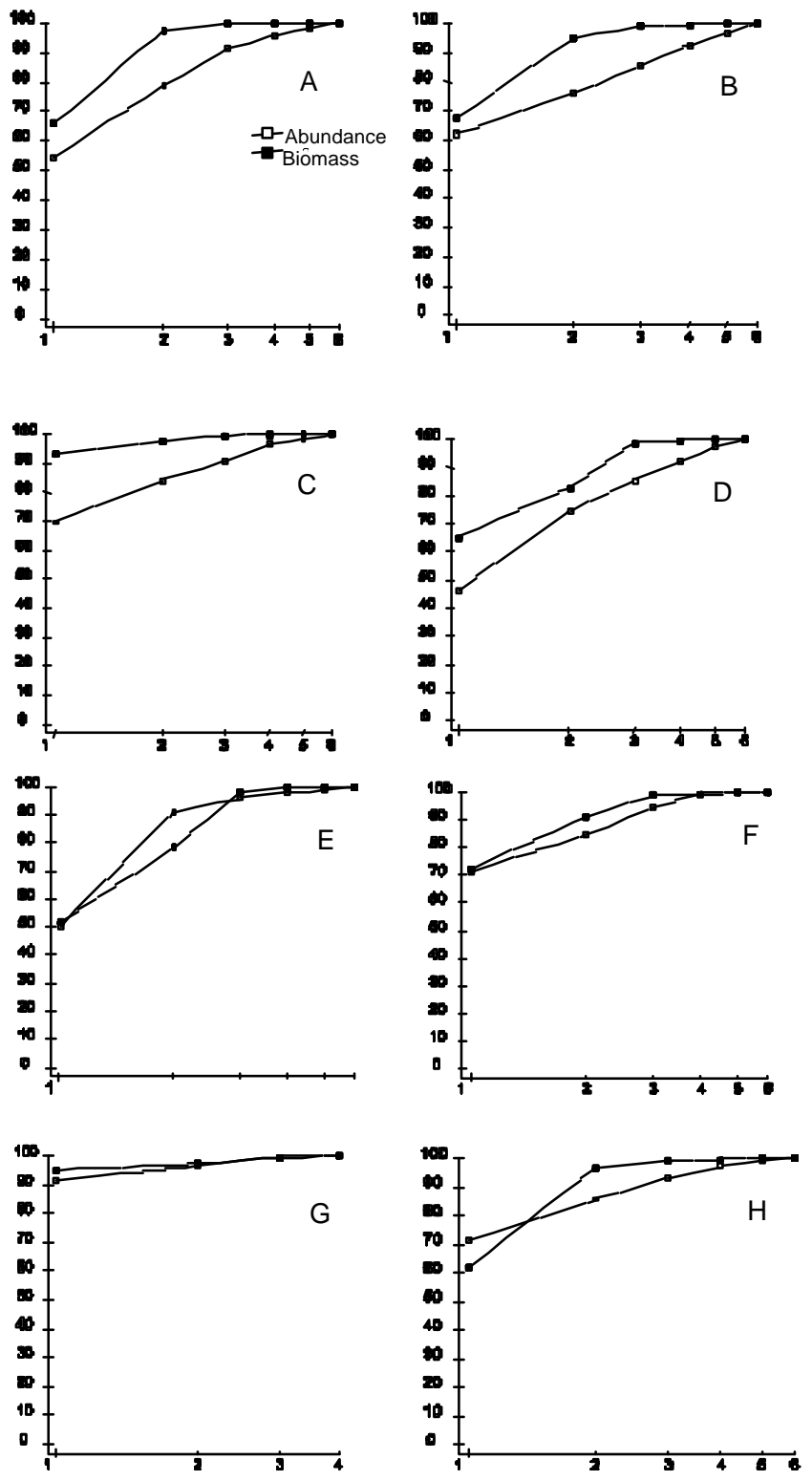

Figure 4. ABC curves applied to sites E1 and E2. (A) Site E1 in winter. (B) Site E1 in spring. (C) Site E1 in summer. (D) Site E1 in autumn. (E) Site E2 in winter. (F) Site E2 in spring. (G) Site E2 in summer. (H) Site E2 in autumn.

Figura 4. Curvas ABC aplicadas a los sitios E1 y E2. (A) Sitio E1 en invierno. (B) Sitio E1 en primavera. (C) Sitio E1 en verano. (D) Sitio E1 en otoño. (E) Sitio E2 en invierno. (F) Sitio E2 en primavera. (G) Sitio E2 en verano. (H) Sitio E2 en otoño. 

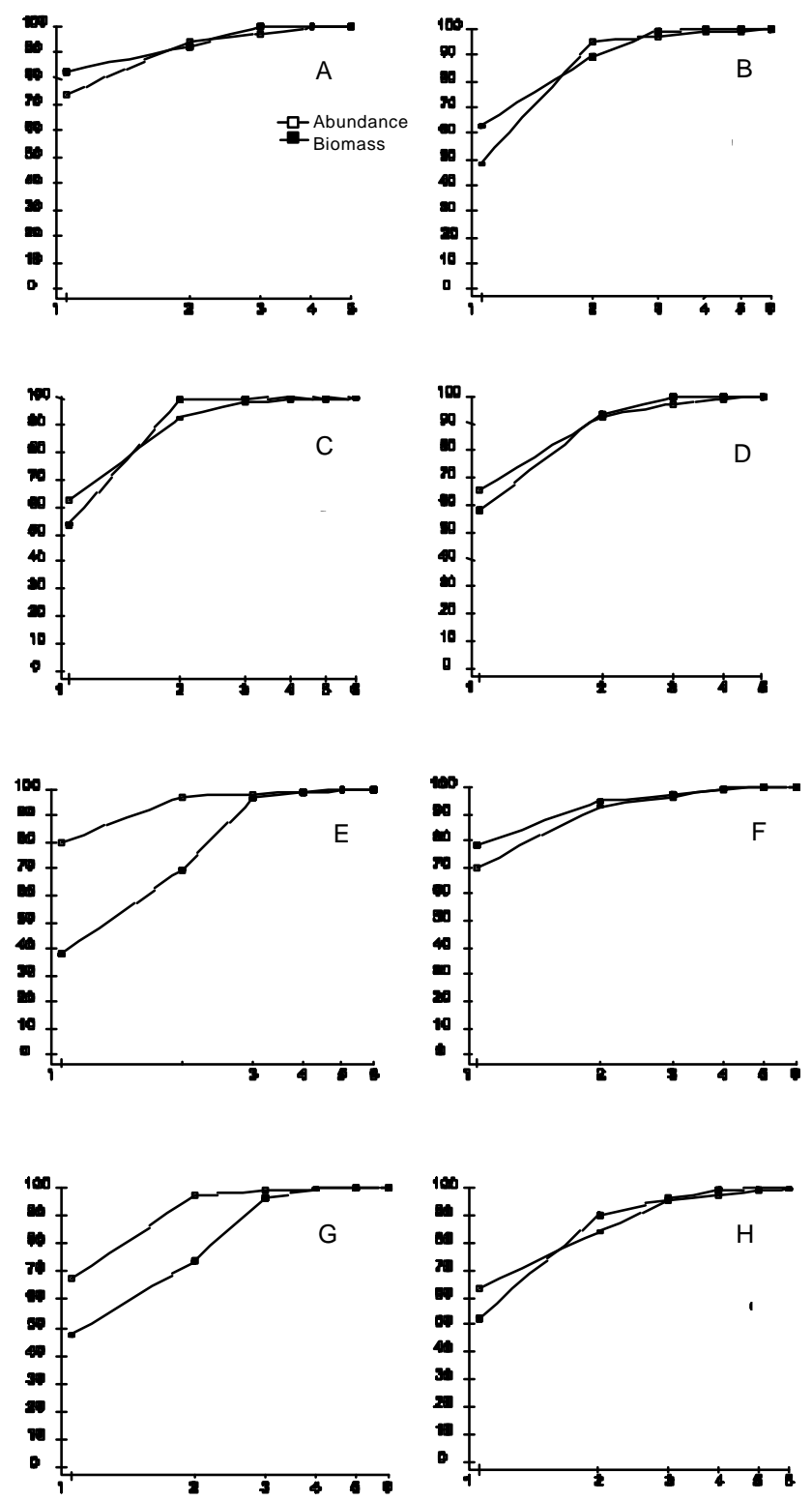

Figure 5. ABC curves applied to sites E3 and E4. (A) Site E3 in winter. (B) Site E3 in spring. (C) Site E3 in summer. (D) Site E3 in autumn. (E) Site E4 in winter. (F) Site E4 in spring. (G) Site E4 in summer. (H) Site E4 in autumn.

Figura 5. Curvas ABC aplicadas a los sitios E3 y E4. (A) Sitio E3 en invierno. (B) Sitio E3 en primavera. (C) Sitio E3 en verano. (D) Sitio E3 en otoño. (E) Sitio E4 en invierno. (F) Sitio E4 en primavera. (G) Sitio E4 en verano. (H) Sitio E4 en otoño. 
environmental factors. Temporal variation in this structure could not obscure the spatial distribution over time.

The community structure of rocky shores is determined by complex interactions of biotic and abiotic factors. Biotic effects can be complex, ranging from grazing effects (Dayton, 1975) and predator/prey interactions (Menge and Sutherland, 1976), to competition for space (Paine, 1971). However, biotic effects take place within a basic physical framework. The effect of wave action has been studied on single trophic compartments such as algae (Kingsbury, 1962), but its influence on the trophic structure of intertidal assemblages has only scarcely been examined (MacLachlan et al., 1981; McQuaid and Branch, 1985). The balance among different trophic compartments of mollusc assemblages on rocky shores may be largely controlled by the degree of wave exposure and type of substrate, as shown in this study. In fact, within the intertidal zone of the study area, the two habitat types distinguished correspond to two main feeding habitats used by different trophic groups: (1a) site E1, a strongly sloping exposed area, covered by crustose, brown and filamentous algae, mainly occupied by suspension-feeders; (1b) site E2, an exposed rocky area, with a gentle slope, dominated by suspension-feeders and herbivores; (2) rocky shores E3 and E4, close to the sand platform covered by brown algae, mostly occupied by herbivores and carnivores, respectively, with a relatively high contribution of suspension-feeders, which occurred most abundantly in exposed areas. A greater abundance of suspension-feeders in exposed intertidal areas has been reported previously (Stephenson and Stephenson, 1972; McQuaid and Branch, 1984, 1985). Our data partially agree with the results obtained by other authors, such as Hawkins and Hartnoll (1983), McQuaid and Branch (1985), and Hily and Jean (1997), who indicated that wave-exposed shores are
E3 y E4, mientras que E1 no presentó variación en una escala temporal (fig. 4A-D). Las curvas mostraron un cambio en la estructura trófica del sitio E2 durante el invierno, el cual se estabilizó en primavera y otoño (fig. 4E-H). El sitio E3 presentó una situación de inestabilidad durante todo el año (fig. 5A-D). El sitio E4 fue muy inestable en invierno, estabilizándose ligeramente en primavera y cambiando de nuevo desde el verano hasta el otoño (fig. 5E-H).

\section{DISCUSIÓN}

\section{Estructura trófica espacial}

El estudio de la estructura trófica aportó evidencias de una clara distribución espacial en la Bahía de Mazatlán. La estructura trófica de las asociaciones en cada sitio de muestreo pareció definirse con base en una serie de factores ambientales locales. La variación temporal de esta estructura trófica no pudo oscurecer la distribución espacial en el tiempo.

La estructura de la comunidad en sustratos rocosos se determina por interacciones complejas entre los factores bióticos y abióticos. Los efectos bióticos pueden ser complejos, variando desde efectos de pastoreo (Dayton, 1975) e interacciones carnívoro/presa (Menge y Sutherland, 1976), hasta competición por el espacio (Paine, 1971). Sin embargo, los factores bióticos actúan sobre un marco previamente establecido por el ambiente físico. Se ha realizado una investigación extensiva de los efectos de la acción de las olas sobre cada especie y también sobre compartimentos tróficos, tales como las algas (Kingsbury, 1962), pero la influencia de estos efectos sobre la estructura trófica de las comunidades intermareales ha sido rara vez examinada (MacLachlan et al., 1981; McQuaid y Branch, 1985). El balance entre los diferentes compartimentos tróficos en las asociaciones de moluscos de sustratos rocosos de este estudio pudo haber estado 
Ciencias Marinas, Vol. 27, No. 2, 2001

dominated by suspension-feeders and sheltered areas are dominated by algae and herbivores. In this study, however, macroalgae dominated in exposed areas (E1, E2) and herbivores dominated in sheltered areas (E3, E4). However, this pattern was due to the fact that most of the herbivorous species that dominated in sheltered areas were microgastropods (e.g., Barleeia alderi, Eulithidium perforata), which feed on microalgae and diatoms.

Furthermore, the type of substrate is an important factor controlling the distribution of organisms (Littler et al., 1983; Airoldi and Virgilio, 1998), since it could have an impoverishing effect on sessile forms (suspensionfeeders) in unstable areas that receive moderate loads of sand (sites E3 and E4). In fact, these sites presented a decrease in sessile forms, being dominated by motile herbivorous and carnivorous organisms.

On the other hand, trophic group complexity was higher at sites E1 and E4 than at sites E2 and E3. Habitat complexity is associated with the increase of trophic groups (Gambi and Giangrande, 1985), and with a more important role of suspension-feeders and carnivores (Bianchi and Morri, 1985). Trophic complexity decreased especially at site E3, where carnivores and suspension-feeders were less represented. Moreover, this parameter fluctuated temporally, with a concomitant increase in winter (table 2), except at E3, where it occurred in autumn (higher carnivore and suspensionfeeder biomass values). In this sense, the trophic complexity and spatio-temporal heterogeneity of the assemblages depend on the particular life-history of the species involved (Polis et al., 1996).

\section{Temporal changes in the trophic structure}

The trend of the biomass to increase in winter and to decrease through summer has already been reported (Pinedo et al., 1997). controlado principalmente por el grado de exposición al oleaje y por el tipo de sustrato. Así, dentro de la zona intermareal del área de estudio, se pudieron distinguir dos tipos de hábitats que se correspondieron con dos principales tipos de hábitats de alimentación de diferentes grupos tróficos: (1a) sitio E1, un área expuesta de fuerte pendiente cubierta por algas incrustantes, algas pardas y filamentosas, que estuvo dominada principalmente por suspensívoros; (1b) sitio E2, un área rocosa expuesta con suave pendiente, la cual estuvo dominada por suspensívoros y herbívoros; (2) sustratos rocosos E3 y E4, próximos a una plataforma arenosa cubierta principalmente por algas pardas, que estuvieron mayormente ocupados por hebívoros y carnívoros, respectivamente, con una fuerte contribución de suspensívoros, aunque dominaron en áreas expuestas. Una mayor abundancia de suspensívoros en áreas intermareales expuestas ha sido observada por algunos autores (Stephenson y Stephenson, 1972; McQuaid y Branch, 1984, 1985). Nuestros datos coinciden parcialmente con los resultados obtenidos por otros autores como Hawkins y Hartnoll (1983), McQuaid y Branch (1985) y Hily y Jean (1997), quienes indicaron que los sustratos expuestos están dominados por suspensívoros y las zonas protegidas están dominadas por algas y herbívoros. En este estudio, sin embargo, las algas dominaron en áreas expuestas (E1, E2) y los herbívoros predominaron en áreas protegidas (E3, E4). Sin embargo, este patrón pudo deberse a que la mayor parte de las especies herbívoras que dominaron las zonas protegidas fueron microgasterópodos (e.g., Barleeia alderi, Euthilidium perforata), que se alimentan de microalgas y diatomeas.

Asimismo, el tipo de sustrato es un factor importante a la hora de controlar la distribución de los organismos (Littler et al., 1983; Airoldi y Virgilio, 1998), ya que éste podría tener un efecto de empobrecimiento sobre las formas sésiles (suspensívoros) en áreas inestables que 
Olabarria et al.: Trophic structure of molluscs on a rocky shore in the tropical Pacific

However, this trend is contrary to the observations of Meire and Dereu (1990) and Platell and Potter (1996), who reported maximum mollusc biomass in spring and summer. Although environmental variables could be modulating temporal changes influencing the assemblage's functional organization, seasonality could be mainly related to recruitment events (high increase in density, followed by high mortality affecting most recruits).

The ABC curves showed a stable assemblage at site E1, while sites E2, E3 and E4 changed throughout the year, except for winter and summer at E4. The sites and functional communities that were assessed as unstable by the $\mathrm{ABC}$ curves indicate a general fluctuation and instability in environmental conditions on the intertidal shores. However, these results did not reflect the real situation at the different sites. Thus, high numbers of small species like Fartulum sp., Barleeia alderi and Caecum compactum, and paucity of species at some sites (e.g., E2 in summer) can be misleading (Roth and Wilson, 1998). On the other hand, the $\mathrm{ABC}$ method may be influenced by recruitment events. All these factors could confound the ABC curves (e.g., juveniles would be equivalent $r$-selected species or opportunistic, numerically dominant yet not representing a large proportion of the assemblage biomass), in such a way that the abundance curve would lie above the biomass curve (Dauvin and Ibanez, 1986; Beukema, 1988; Lardicci and Rossi, 1998). Therefore, depending on the species involved, the results of the $\mathrm{ABC}$ curves may only sometimes be consistent.

The study of the local trophic structure provided evidence of a clear spatial distribution at Mazatlán Bay. The trophic structure of the assemblages at each sampling site seemed to be defined by a particular set of local environmental factors. The balance among different trophic compartments of mollusc communities may be largely controlled by the degree of reciben cantidades moderadas de arena (sitios E3 y E4). De hecho, estos sitios presentaron una disminución de formas sésiles, siendo dominados por organismos móviles, tales como herbívoros y carnívoros.

Por otro lado, la complejidad de grupo trófico fue mayor en los sitios E1 y E4 que en los sitios E2 y E3. La complejidad está asociada con el incremento de grupos tróficos (Gambi y Giangrande, 1985), y con un papel más importante de los suspensívoros y de los carnívoros (Bianchi y Morri, 1985). La complejidad trófica disminuyó en el sitio E3, donde los carnívoros y suspensívoros estuvieron menos representados. Asimismo, este parámetro fluctuó temporalmente, con un importante incremento en invierno (tabla 2), excepto en E3 que lo tuvo en otoño (biomasa de carnívoros y suspensívoros más alta). En este sentido, la complejidad trófica y la heterogeneidad espacial de las asociaciones dependen de la historia de vida de cada especie particular (Polis et al., 1996).

\section{Cambios temporales en la estructura trófica}

La tendencia de la biomasa a incrementar en invierno y disminuir a lo largo del verano ya ha sido observada por algunos autores (Pinedo et al., 1997). Sin embargo, esta observación no coincide con las realizadas por Meire y Dereu (1990) y Platell y Potter (1996), quienes detectaron la máxima biomasa de moluscos en primavera y verano. Aunque las variables ambientales podrían estar contribuyendo a modular cambios temporales que influyen en la organización de la comunidad, la estacionalidad podría estar relacionada principalmente con fenómenos de reclutamiento (alto incremento de densidad, seguido por una alta mortalidad afectando a la mayoría de los reclutas).

Las curvas $\mathrm{ABC}$ mostraron una asociación estable en el sitio E1, mientras que los sitios E2, E3 y E4 presentaron cambios en su 
Ciencias Marinas, Vol. 27, No. 2, 2001

wave exposure (see table 3) and type of substrate (e.g., sand deposition, slope). On the other hand, the $\mathrm{ABC}$ curves showed an unstable situation at sites E2, E3 and E4 over time. However, these changes may be related to biological factors, such as recruitment and the presence of many small species.

\section{ACKNOWLEDGEMENTS}

This research was supported by CONACYT (project 091 Pñ-1297). We thank Alma Velasco for translating this manuscript.

\section{REFERENCES}

Airoldi, L. and Virgilio, M. (1998). Responses of turf-forming algae to spatial variations in the deposition of sediments. Mar. Ecol. Prog. Ser., 165: 271-282.

Andrew, N.L and Choat, J.H. (1985). Habitat-related differences in the survivorship and growth of juvenile sea urchins. Mar. Ecol. Prog. Ser., 27: 155-161.

Beukema, J.J. (1988). An evaluation of the ABCmethod (abundance/biomass comparison) as applied to macrozoobenthic communities living on tidal flats in the Dutch Wadden Sea. Mar. Biol., 99: 425-433.

Bianchi, C.N. and Morri, C. (1985). I policheti come descrittori della struttura trofica degli ecosistemi marini. Oebalia, 11: 203-214.

Bray, J.R. and Curtis, J.T. (1957). An ordination of the upland forest communities of southern Wisconsin. Ecol. Monogr., 27: 325-349.

Clarke, K.R. (1990). Comparisons of dominance curves. J. Exp. Mar. Biol. Ecol., 138: 143-157.

Commito, J.A. and Ambrose, W.G. (1985). Predatory infauna and trophic complexity in soft-bottoms. In: P.E. Gibbs (ed.), Proc. 19th European Marine Biology Symp. Cambridge Univ. Press, Cambridge, pp. 323-333.

Connell, J.B. (1961). The influence of interspecific competition and other factors on the distribution of the barnacle Chthamalus stellatus. Ecology, 42: 710-723. estructura a lo largo del año, excepto en el caso del sitio E4 en invierno y verano. El alto número de sitios y comunidades funcionales que fueron caracterizados como inestables por las curvas $\mathrm{ABC}$, indican una fluctuación general e inestabilidad en las condiciones ambientales de los sustratos intermareales. Sin embargo, estos resultados no reflejaron la situación real en los diferentes sitios. Así, un alto número de pequeñas especies, tales como Fartulum sp., Barleeia alderi y Caecum compactum, y la escasez de especies en algunos sitios (E2 en verano) pueden confundir los resultados (Roth y Wilson, 1998). Por otro lado, el método ABC podría estar influido por fenómenos de reclutamiento. Todos estos factores podrían confundir las curvas $\mathrm{ABC}$ (e.g., juveniles pueden ser equivalentes a estrategias- $r$ o especies oportunistas, numéricamente dominantes, pero que no representan una parte importante de la biomasa de la asociación), de forma que la curva de la abundancia se situaría por encima de la de biomasa (Dauvin e Ibanez, 1986; Beukema, 1988; Lardicci y Rossi, 1998). Es decir, dependiendo de las especies involucradas, los resultados de las curvas $\mathrm{ABC}$ pueden ser consistentes solamente en determinadas ocasiones.

El estudio de la estructura trófica aportó evidencias de una clara distribución espacial en la Bahía de Mazatlán. La estructura trófica de las asociaciones en cada sitio de muestreo pareció estar definida por una serie de factores ambientales locales particulares. El balance entre los diferentes compartimentos tróficos en las asociaciones de moluscos podría estar fuertemente controlado por el grado de exposición al oleaje (tabla 3 ) y por el tipo de sustrato (e.g., deposición de arena, pendiente). Por otro lado, las curvas $\mathrm{ABC}$ mostraron una situación inestable a lo largo del tiempo en los sitios E2, E3 y E4. Sin embargo, estos cambios pudieron estar relacionados con factores biológicos, tales como reclutamiento y la presencia de un alto número de pequeñas especies. 
Cornet, M. (1985). Recherches sur l'écologie des mollusques bivalves du plateau continental sud-Gascogne. Ann. Inst. Océanogr., Paris, 61: $59-74$.

Cornet, M. (1986). Évolutions des paramétres de structure des populations de mollusques bivalves des fonds à Abra alba au large du bassin d'Arcachon. Vie et Milieu, 36: 15-25.

Dauvin, J.C. and Ibanez, F. (1986). Variations à long-terme (1977-1985) du peuplement des sables de la Pierre Noire (Baie de Morlaix, Manche Occidentale): Analyse statistique de l'évolution structurale. Hydrobiologia, 142: 171-186.

Day, R.W. and Quinn, G.P. (1989). Comparisons of treatments after an analysis of variance in ecology. Ecol. Monogr., 59: 433-463.

Dayton, P.K. (1975). Experimental evaluation of ecological dominance in a rocky intertidal algal community. Ecol. Monogr., 45: 137-159.

Estacio, F.J., Adiego, E., Carballo, J.L., SánchezMoyano, E., Izquierdo, J.J. and García-Gómez, J.C. (1999). Interpreting temporal disturbances in an estuarine benthic community under combined anthropogenic and climatic effects. J. Coast. Res., 15 (1): 155-167.

Field, J.G., Clarke, K.R. and Warwick, R.M. (1982). A practical strategy for analysing multispecies distribution patterns. Mar. Ecol. Prog. Ser., 8: $37-52$.

Gagnon, J.M. and Haedrich, R.L. (1991). A functional approach to the study of Labrador/ Newfoundland shelf macrofauna. Cont. Shelf Res., 11: 963-976.

Gambi, M.C. and Giangrande, A. (1985). Analisi della struttura trofica del popolamento dei policheiti nei fondi mobili di due arre del Mar Tirreno. Oebalia, 11: 223-240.

Gray, J.S. (1974). Animal-sediment relationships. Oceanogr. Mar. Biol. Ann. Rev., 12: 223-261.

Gray, J.S. (1981). The Ecology of Marine Sediments. Cambridge Univ. Press, Cambridge, $185 \mathrm{pp}$.

Hawkins, S.J. and Hartnoll, R.G. (1983). Grazing of intertidal algae by marine invertebrates. Oceanogr. Mar. Biol. Ann. Rev., 21: 195-282.

Hily, C. and Jean, F. (1997). Macrobenthic biodiversity in intertidal habitats of the Iroise

\section{AGRADECIMIENTOS}

Este trabajo fue realizado con apoyo del CONACYT (proyecto 091 Pñ-1297). Agradecemos a Alma Velasco la traducción de este manuscrito.

biosphere reserve (Brittany, France). J. Mar. Biol. Assoc. UK, 77: 311-323.

Keen, M. (1971). Sea Shells of Tropical West America. Stanford Univ. Press, Stanford, California, 1064 pp.

Kingsbury, J.M. (1962). The effect of waves on the composition of a population of attached marine algae. Bull. Torrey Bot. Club, 89: 143-160.

Lardicci, C. and Rossi, F. (1998). Detection of stress on macrozoobenthos: Evaluation of some methods in a coastal Mediterranean lagoon. Mar.Environ. Res., 45: 367-386.

Lewis, J.R. (1964). Ecology of Rocky Shores. English Univ. Press, London, 543 pp.

Littler, M.M., Martz, D.R. and Littler, D.S. (1983). Effects of recurrent sand deposition on rocky intertidal organisms: Importance of substrate heterogeneity in a fluctuating environment. Mar.Ecol. Prog. Ser., 11: 129-139.

McLachlan, A., Lombard, H.W. and Louwrens, S. (1981). Trophic structure and biomass distribution on two East Cape rocky shores. S.Afr. J. Zool., 12: 405-437.

McQuaid, C.D. and Branch, G.M. (1984). The influence of sea temperature, substratum and wave exposure on rocky intertidal communities: An analysis of faunal and floral biomass. Mar.Ecol. Prog. Ser., 19: 145-151.

McQuaid, C.D. and Branch, G.M. (1985). Trophic structure of rocky intertidal communities: Response to wave action and implications for energy flow. Mar. Ecol. Prog. Ser., 22: 153-161.

Meire, P.M. and Dereu, J. (1990). Use of the abundance/biomass comparison method for detecting environmental stress: Some considerations based on intertidal macrozoobenthos and bird communities. J. Appl. Ecol., 27: 210-223. 
Ciencias Marinas, Vol. 27, No. 2, 2001

Menge, B.A. and Sutherland, J.P. (1976). Species diversity gradients: Synthesis of the roles of predation, competition and temporal heterogeneity. Am. Nat., 110: 351-369.

Odum, U.P. (1971). Fundamentals of Ecology. Saunders Co., Philadelphia, London, Toronto, $574 \mathrm{pp}$.

Paine, R.T. (1971). A short-term experimental investigation of resource partitioning in a New Zealand rocky intertidal habitat. Ecology, 52: 1096-1106.

Pinedo, S., Sardá, R. and Martin, D. (1997). Comparative study of the trophic structure of soft-bottom assemblages in the Bay of Blanes (western Mediterranean Sea). Bull. Mar. Sci., 60: 529-542.

Platell, M.E. and Potter, I.C. (1996). Macrobenthos of a seasonally closed estuary. J. Mar. Biol. Assoc. UK, 76: 1-21.

Polis, G.A, Holt, R.D., Menge, B.A. and Winemiller, K.O. (1996). Time, space, and life history: Influences on food webs. In: G.A. Polis and K.O. Winemiller (eds.), Food Webs. Chapman \& Hall, pp. 435-461.

Reguero, M., García-Cubas, A. and Zúñiga, G. (1991). Moluscos de la Laguna Tampamachoco, Veracruz, México: Sistemática y ecología. An. Inst. Cienc. Mar Limnol., UNAM, 18: 289-328.

Reguero, M., García-Cubas, A. and Zúñiga, G. (1993). Moluscos del complejo lagunar LargaRedonda-Mandinga, Veracruz, México: Sistemática y ecología. Hidrobiológica, 3: 41-70.

Roth, S. and Wilson, J. (1998). Functional analysis by trophic guilds of macrobenthic community structure in Dublin Bay, Ireland. J. Exp. Mar. Biol. Ecol., 222: 195-217.
Stephenson, T.A. and Stephenson, A. (1972). Life between Tidemarks on Rocky Shores. W.H. Freeman, San Francisco, 231 pp.

Underwood, A.J. and Denley, E.J. (1984). Paradigms, explanations and generalizations in models for the structure of intertidal communities on rocky shores. In: D.R. Strong, D. Simberloff Jr., L.G. Abele and A.B. Thistle (eds.), Ecological Communities: Conceptual Issues and the Evidence. Princeton Univ. Press, Princeton, pp. 151-180.

Underwood, A.J. and Chapman, M.J. (1999). Problems and practical solutions for quantitative assessment of biodiversity of invertebrates in coastal habitats. In: W. Ponder and D. Lunney (eds.), The Other 99\%. The Conservation and Biodiversity of Invertebrates. Royal Society of New South Wales, Mosman, pp. 19-25.

Walker, A.J.M. and Rees, E.I.S. (1979). Benthic ecology of Dublin Bay in relation to sludge dumping: Fauna. Irish Fish. Invest. Ser. B, 22: $1-59$.

Warwick, R.M. (1982). The partitioning of secondary production among species in benthic communities. Neth. J. Sea Res., 16: 1-17.

Warwick, R.M. and Ruswahyuni, R. (1987). Comparative study of the structure of some tropical and temperate marine soft-bottom macrobenthic communities. Mar. Biol., 95: 641-649.

Warwick, R.M., Pearson, T.H. and Ruswahyuni, R. (1987). Detection of pollution effects on marine macrobenthos: Further evaluation of the species abundance-biomass method. Mar. Biol., 95: 193-200.

Whitlatch, R.B. and Obrebski, S. (1980). Feeding selectivity and coexistence in two depositfeeding gastropods. Mar. Biol., 58: 219-225. 